\title{
Integral field spectroscopy with SINFONI of VVDS galaxies ${ }^{\star}$
}

\section{The mass-metallicity relation at $1.2<z<1.6$}

\author{
J. Queyrel ${ }^{1}$, T. Contini ${ }^{1}$, E. Pérez-Montero ${ }^{1,3}$, B. Garilli ${ }^{4}$, O. Le Fèvre ${ }^{2}$, M. Kissler-Patig ${ }^{6}$, B. Epinat ${ }^{1}$, D. Vergani ${ }^{5}$, \\ L. Tresse ${ }^{2}$, P. Amram ${ }^{2}$, and M. Lemoine-Busserolle \\ ${ }^{1}$ Laboratoire d'Astrophysique de Toulouse-Tarbes, Université de Toulouse, CNRS, 14 Avenue Édouard Belin, 31400 Toulouse, \\ France \\ e-mail: jqueyrel@ast.obs-mip.fr \\ ${ }^{2}$ Laboratoire d'Astrophysique de Marseille, Université de Provence, CNRS, 38 rue Frédéric Joliot-Curie, 13388 Marseille Cedex 13, \\ France \\ 3 Instituto de Astrofísica de Andalucía - CSIC Apdo. 300418080 Granada, Spain \\ ${ }^{4}$ IASF-INAF, Via Bassini 15, 20133 Milano, Italy \\ 5 INAF - Osservatorio Astronomico di Bologna, via Ranzani 1, 40127 Bologna, Italy \\ ${ }^{6}$ ESO, Karl-Schwarzschild-Str.2, 85748 Garching b. München, Germany \\ 7 Oxford Physics, University of Oxford, Keble Road, Oxford OX13RH, UK
}

Received 6 March 2009 / Accepted 7 August 2009

\section{ABSTRACT}

\begin{abstract}
Aims. This work aims to provide a first insight into the mass-metallicity $(M Z)$ relation of star-forming galaxies at redshift $z \sim 1.4$. To reach this goal, we present a first set of nine VVDS (VIMOS VLT Deep Survey) galaxies observed with the NIR integral-field spectrograph SINFONI on the VLT.

Methods. Oxygen abundances are derived from empirical indicators based on the ratio between strong nebular emission-lines (H $\alpha$, [N II]6584 Å and [S II]6717, 6731 A). Stellar masses are deduced from SED fitting with Charlot \& Bruzual (2003) population synthesis models, and star formation rates are derived from [O II $] 3727 \AA$ and $\mathrm{H} \alpha$ emission-line luminosities.

Results. We find a typical shift of $0.2-0.4$ dex towards lower metallicities for the $z \sim 1.4$ galaxies, compared to the $M Z$-relation in the local universe as derived from SDSS data. However, this small sample does not show any clear correlation between stellar mass and metallicity, unlike other larger samples at different redshift $(z \sim 0, z \sim 0.7$, and $z \sim 2)$. Indeed, our galaxies lie just under the relation at $z \sim 2$, and show a small trend for more massive galaxies to be more metallic $(\sim 0.1$ logarithmic slope). There are two possible explanations to account for these observations. First, our galaxies present higher specific star formation rates when compared to the global VVDS sample which could explain the particularly low metallicity of these galaxies as already shown in the SDSS sample. Second, inflow of metal-poor gas due to tidal interactions could also explain the low metallicity of these galaxies as three of these nine galaxies show clear signatures of merging in their velocity fields. Finally, we find that the metallicity of four galaxies is lower by $\sim 0.2$ to 0.4 dex if we take into account the N/O abundance ratio in their metallicity estimate.
\end{abstract}

Key words. galaxies: abundances - Galaxy: evolution - galaxies: high-redshift

\section{Introduction}

The abundance of heavy elements in galaxies reflects the past history of star formation and the effects of gas exchanges (inflows and/or outflows) with the intergalactic medium. A characterization of the evolution of chemical abundances for galaxies of different masses is therefore essential to a complete model of galaxy formation that includes the physics of baryons (see e.g. De Lucia et al. 2004; de Rossi et al. 2007; Finlator \& Davé 2008).

First discovered for irregular galaxies (Lequeux et al. 1979), the mass-metallicity (hereafter $M Z$ ) relation has been investigated intensively (Brodie \& Huchra 1981; Skillman et al. 1989; Zaritsky et al. 1994; Garnett et al. 1997, among others) and is

^ Based on observations collected at the European Southern Observatory (ESO) Very Large Telescope, Paranal, Chile, as part of the Programs 75.A-0318, 78.A-0177, and 070.A-9007. now well established in the local universe $(z<0.2)$ thanks to the analysis of data from large spectroscopic surveys such as the Sloan Digital Sky Survey (SDSS; Tremonti et al. 2004; Gallazzi et al. 2005; Panter et al. 2008) and the 2 degree Field Galaxy Redshift Survey (2dFGRS; Lamareille et al. 2004). The $M Z$ relation for local galaxies is steep for masses $\leq 10^{10.5} M_{\odot}$ and flattens at higher masses suggesting that the $M Z$ relation is mainly driven by the decrease of metal loss when stellar mass increases. These trends observed by Tremonti et al. (2004) and Lamareille et al. (2004) have been shown to extend to much lower galaxy masses $\left(M<10^{9} M_{\odot}\right.$; e.g. Lee et al. 2006; Saviane et al. 2008). Recent studies focused on the dependence of the $M Z$ relation of SDSS galaxies with environment on small scales (Michel-Dansac et al. 2008; Ellison et al. 2008b) and on larger scales (Mouhcine et al. 2007; Cooper et al. 2008) and their star formation rate and galaxy sizes (Ellison et al. 2008a).

The evolution of the $M Z$ relation on cosmological timescales is now predicted by semi-analytical models of galaxy formation. 
Table 1. Properties of the nine galaxies observed with SINFONI.

\begin{tabular}{ccccccc}
\hline \hline Galaxy & $\operatorname{RA}(2000)$ & $\operatorname{Dec}(2000)$ & $\begin{array}{c}t_{\text {exp }}(\text { band })^{1} \\
\text { [hours] }\end{array}$ & $I_{A B}$ & $z_{\text {VIMOs }}{ }^{2}$ & $z_{\text {SINFONI }}{ }^{3}$ \\
\hline VVDS020116027 & $02: 25: 51.2$ & $-04: 45: 07.5$ & $1.7(\mathrm{H})$ & 22.87 & 1.5259 & 1.5302 \\
VVDS020182331 & $02: 26: 44.3$ & $-04: 35: 51.9$ & $3(\mathrm{H})$ & 22.73 & 1.2286 & 1.2283 \\
VVDS020147106 & $02: 26: 45.5$ & $-04: 40: 46.9$ & $2(\mathrm{H})$ & 22.50 & 1.5174 & 1.5195 \\
VVDS020261328 & $02: 27: 11.1$ & $-04: 25: 30.7$ & $1(\mathrm{H})$ & 23.90 & 1.5291 & 1.5289 \\
VVDS220596913 & $22: 14: 29.2$ & $+00: 22: 15.7$ & $1.75(\mathrm{H})$ & 21.84 & 1.2667 & 1.2662 \\
VVDS220584167 & $22: 15: 22.8$ & $+00: 18: 47.5$ & $1.75(\mathrm{H}), 1(\mathrm{~J})$ & 22.04 & 1.4637 & 1.4659 \\
VVDS220544103 & $22: 15: 25.5$ & $+00: 06: 40.0$ & $1(\mathrm{H}), 1(\mathrm{~J})$ & 22.47 & 1.3970 & 1.3966 \\
VVDS220015726 & $22: 15: 42.5$ & $+00: 29: 04.9$ & $2(\mathrm{H})$ & 22.47 & 1.3091 & 1.2930 \\
VVDS220014252 & $22: 17: 45.5$ & $+00: 28: 40.3$ & $2(\mathrm{H})$ & 22.10 & 1.3097 & 1.3101 \\
\hline
\end{tabular}

${ }^{1}$ Exposure time corresponding to the effective time spent on the source in each band $(H$ or $J) ;{ }^{2}$ redshift based on the VIMOS spectrum; ${ }^{3}$ redshift estimate from the position of $\mathrm{H} \alpha$ in SINFONI spectra.

Reliable observational estimates of the $M Z$ relation of galaxies at different epochs (and hence different redshifts) may thus provide important constraints on galaxy evolution scenarios. At increasing redshifts, as the strong rest-frame optical emission lines shift into the near-infrared (NIR) window, metallicities are typically based on smaller subsets of strong emission lines, through the use of empirically calibrated abundance indicators. Much progress has been made recently in assembling large samples of star-forming galaxies with abundance measurements at both intermediate redshifts $(z<1$; Savaglio et al. 2005; Maier et al. 2005; Liang et al. 2006; Lamareille et al. 2006, 2009; Pérez-Montero et al. 2009; Cowie \& Barger 2008) and at $z$ > 2 (Erb et al. 2006; Maiolino et al. 2008; Hayashi et al. 2009; Mannucci et al. 2009). However, chemical abundance measurements are only available for a limited number of galaxies at $z \sim 1-2$ (Shapley et al. 2005; Maier et al. 2006; Liu et al. 2008). The rather unexplored $1<z<2$ redshift regime is one of particular importance in the history of the universe corresponding to i) the peak of star formation rate, and hence metal production, for the universe as a whole (see e.g. Lilly et al. 1996; Madau et al. 1996; Pérez-González et al. 2005; Tresse et al. 2007); ii) the buildup of a significant fraction of the stellar mass in the universe (e.g. Drory et al. 2005; Arnouts et al. 2007; Pozzetti et al. 2007); and iii) the emergence of the Hubble sequence of disks and spheroids.

We have undertaken an large observing program at ESOVLT aimed at probing the mass assembly and metallicity evolution of a representative sample of galaxies at $1<z<2$. The final goal of the MASSIV ${ }^{1}$ (Mass Assembly Survey with SINFONI in VVDS) project is to obtain a detailed description of the mix of dynamical types (rotating disks, spheroids, and mergers) at this epoch and to follow the evolution of fundamental scaling relations, such as the Tully-Fisher and $M Z$ ones, and therefore constrain galaxy evolution scenarios. In this paper we present the first results obtained by such a program focusing on the $M Z$ relation at $z \sim 1.4$. A companion paper (Epinat et al. 2009) is devoted to the kinematical analysis of galaxies at these redshifts using the same data. This work is based on a sample of nine galaxies selected in the VIMOS VLT Deep Survey (VVDS; Le Fèvre et al. 2005) and observed in the NIR with the 3D-spectrograph SINFONI on the ESO/VLT (Eisenhauer et al. 2003) during pilot observing runs. Throughout this paper we

${ }_{1}^{1}$ www.ast.obs-mip.fr/massiv/ assume a standard $\Lambda$-CDM cosmology with $h=0.7, \Omega_{\Lambda}=0.7$, and $\Omega_{\mathrm{m}}=0.3$.

\section{Data description}

\subsection{Observations}

The selection of the sample and the SINFONI observations are fully described in Epinat et al. (2009). For the convenience of the reader, we summarize here the main steps.

We have used the VIMOS VLT Deep Survey to select galaxies with know spectroscopic redshifts in the range $1<z<2$. The VVDS is a complete magnitude selected sample avoiding the biases linked to more crude a priori color selection techniques. It offers the advantage to combine a large sample with a robust selection function and secure spectroscopic redshifts necessary to engage into long single objects integrations with SINFONI being sure to observe the $\mathrm{H} \alpha$ line outside of the $\mathrm{OH}$ sky emission lines.

The nine galaxies studied in this paper have been selected among those showing the strongest [O II] $3727 \AA$ emission line $\left(E W>50 \AA\right.$ and flux $>5 \times 10^{-17} \mathrm{erg}^{-1} \mathrm{~cm}^{-2}$, as measured on VIMOS spectra) for $\mathrm{H} \alpha$ to be easily detected in the near-IR. These criteria for selecting late-type star-forming galaxies have been shown to be very efficient. From the first observing runs, our success rate of selection has been around 85\%: 9 galaxies over 12 observed show strong rest-frame optical emission lines in SINFONI datacubes. The other three galaxies have not been detected with SINFONI for the following reasons. The redshift of one galaxy is such that $\mathrm{H} \alpha$ is falling just in the gap between the $J$ and $H$ bands. For another galaxy, the strength of [O II] $3727 \AA$ was overestimated. New measurements of this emission line show that this galaxy is now outside of the selection box. The third non-detected galaxy had a wrong redshift.

Among the nine VVDS star-forming galaxies studied in this paper, five have been selected in the VVDS-22h wide field (17.5 $\left.\leq I_{A B} \leq 22.5\right)$ and four in the VVDS-02h deep field $\left(17.5 \leq I_{A B} \leq 24.0\right)$. Their basic properties (RA, Dec, redshift, $I$-band magnitude, etc.) are listed in Table 1 . These targets span a redshift range between $1.27 \lesssim z \lesssim 1.53$. The NIR spectroscopic observations were acquired with the 3D-spectrograph SINFONI at ESO-VLT during two 4-nights run on September 5-8, 2005 (ESO run 75.A-0318) and November 12-15, 2006 (ESO run 78.A-0177). SINFONI was used in its seeing-limited mode, with the $0.25^{\prime \prime} \times 0.125^{\prime \prime}$ pixel scales leading to a field-of-view 

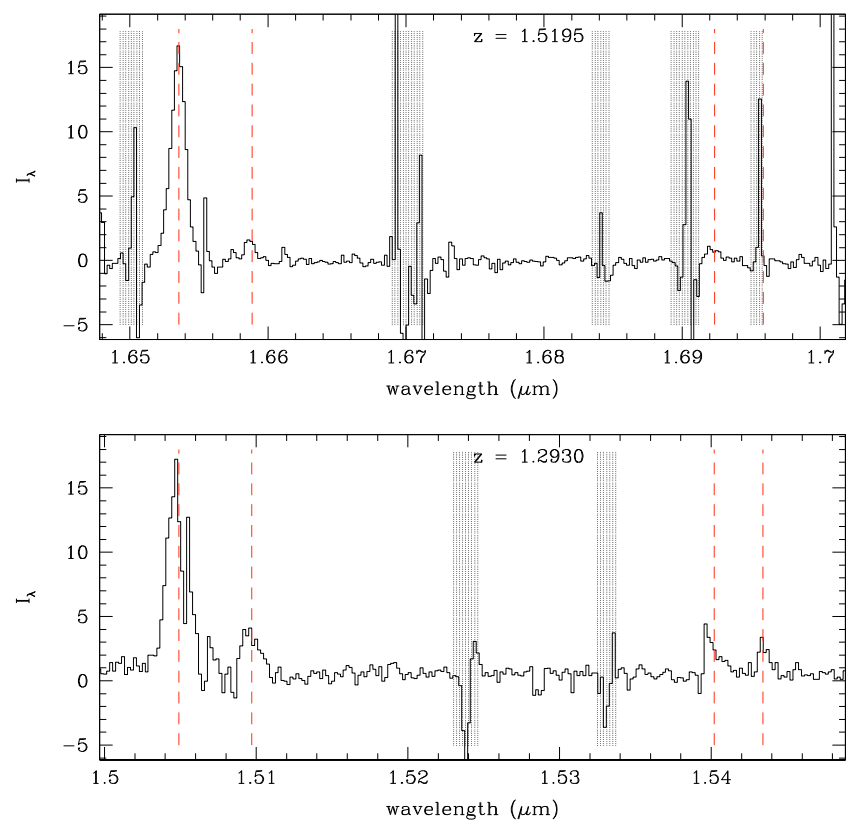

Fig. 1. Examples of 1D spectrum for two galaxies VVDS220015726 (bottom) and VVDS020147106 (top) observed with SINFONI in the $H$ band. The spectral region of interest is centered around the $\mathrm{H} \alpha$, [N II]6584 $\AA$ and [S II]6717, $6731 \AA$ emission lines. The position of these lines is indicated by vertical dotted lines. The other lines with P-cygni profiles visible in the spectra are residuals of OH sky lines subtraction, they are shown within grey zones.

of $8^{\prime \prime} \times 8^{\prime \prime}$, and the $H$-band grism providing a spectral resolution $R \sim 4000$. Note also that two galaxies (VVDS220544103 and VVDS220584167) have also been observed with the $J$-band grism. Conditions were not photometric and the mean seeing was around $0.65^{\prime \prime}$. The total on-source integration times, as well as general information are listed in Table 1. For each Observation Block a hot telluric standard star has been observed. With this star we were able to flux-calibrate our observations with an accuracy of about $20 \%$. Details for data reduction are available in Epinat et al. (2009).

\subsection{Measurement of emission lines}

To perform the spectrophotometric analysis of the reduced datacubes and measure the detected emission lines, we first produced 1D spectra from the 3D data. It has been performed in two steps: first, we produced a 2D pseudo-slit spectrum from the datacube (as if we placed a long-slit over the spatial extent of the object), and then we reduced the 2D-spectrum in a 1D-one, taking care of the spatial extent of the galaxy and of the evolution of its position through the dispersion axis. The first step has been done with the QFitsView software ${ }^{2}$ which is a tool to visualize and analyse datacubes. The second step was performed using the IRAF software, specifically applying the apall task of the noao>twodspec $>$ apextract package. Figure 1 shows examples of 1D spectra for the galaxies VVDS020147106 and VVDS220015726.

At the distance of the sample galaxies, the $\mathrm{H} \alpha$, [N II] $6584 \AA$ and [S II]6717, $6731 \AA$ lines are redshifted into the SINFONI $H$ band. We were thus able to measure these lines in quasi-all our sample galaxies. For two galaxies we had observations in the $J$ band, which enables to detect and measure [O III]4959, $5007 \AA$

2 http://www.mpe.mpg.de/ ott/QFitsView/ and $\mathrm{H} \beta$ lines for VVDS220544103. Unfortunately, the redshift of the second galaxy VVDS220584167 makes the reliable measurement of these lines impossible as they fall at the location of the bright $\mathrm{OH}$-lines. We measured the flux in the different lines fitting a Gaussian to the 1D spectrum of each galaxies (within the splot task in IRAF). Fluxes and errors are obtained with the deblending mode using 100 simulations for the poissonian noise model (see the splot task help for more information). Results are shown in Table 2. Two reasons can account for the impossibility to measure a line: either an $\mathrm{OH}$ sky line falls right upon the line of interest, or the intensity of the line is too low to be detected. Over the nine galaxies, [N II] $6584 \AA$ is not detected for two (VVDS020261328 and VVDS020182331).

The measured values of $\log ([\mathrm{N}$ II $] 6584 / \mathrm{H} \alpha)$ range from -1.3 to -0.64 , which indicates that the sources are star-forming galaxies with at most a low AGN contamination. We also checked for possible X-ray identification of AGN in our sample using the extensive HESEARC archive. Neither XMM nor Chandra source has been identified in the vincinity of our targets further excluding any AGN contamination in our sample. This issue is further discussed in Epinat et al. (2009).

\section{Stellar mass and metallicity estimates}

\subsection{Stellar mass}

The procedure to derive stellar mass of our galaxies is based on the comparison between observations, basically the photometric SED of galaxies, and population synthesis models. More precisely, $M^{\star}$ estimates were obtained with the GOSSIP spectral energy distribution (SED) modeling software (Franzetti et al. 2008). For the SED fitting the multi-band photometric observations available in the VVDS fields were used, including $B V R I$ data from the CFHT, UBVRZs data from the CFHT Legacy Survey, $J$ - and $K$-bands data from SOFI at the NTT and from the UKIDSS survey, and the VVDS-Deep spectra. The stellar population models used for the fit were generated with the $\mathrm{BCO} 3$ population synthesis code (Bruzual \& Charlot 2003), assuming a set of "delayed" star formation histories (see Gavazzi et al. 2002, for details), and a Salpeter IMF. The values and erros bars of the stellar masses in Table 3 are the median of the Probability Distribution Function and its confidence regions. More details can be found in Epinat et al. (2009).

\subsection{Metallicity}

To determine the gas-phase oxygen abundance of the nine VVDS galaxies, we made use of well-known empirical abundance indicators. Indeed, at these redshifts, we only have access to the brightest recombination and forbidden emission lines of the ionized interstellar gas, preventing any accurate metallicity estimate based on faint [O III] auroral lines. Different calibrations or empirical indicators have been proposed over the past 20 years, each of them taking into account different set of emission lines. The most commonly used, but which has the drawback of being degenerated, is certainly $R_{23}=([\mathrm{O}$ II $] 3727+$ $[\mathrm{O}$ III $] 4959,5007) / \mathrm{H} \beta$, first introduced by Pagel et al. (1979), and re-calibrated later in many articles (Kewley \& Dopita 2002; Nagao et al. 2006; Yin et al. 2006).

Considering that the nine galaxies have been observed with SINFONI in the $H$ band, we had access to $\mathrm{H} \alpha$ and [N II]6584 A emission-line fluxes for six galaxies (we did not 
Table 2. Emission-line flux (in $10^{-17} \mathrm{erg} \mathrm{s}^{-1} \mathrm{~cm}^{-2}$ ) for the nine galaxies observed with SINFONI.

\begin{tabular}{ccccccccc}
\hline \hline Galaxy & {$[\mathrm{O} \mathrm{II}] 3727^{1}$} & $\mathrm{H} \beta$ & {$[\mathrm{O}$ III $] 4959$} & {$[\mathrm{O} \mathrm{III}] 5007$} & $\mathrm{H} \alpha$ & {$[\mathrm{N} \mathrm{III}] 6584$} & {$[\mathrm{~S} \mathrm{II}] 6717$} & {$[\mathrm{~S} \mathrm{II}] 6731$} \\
\hline VVDS020116027 & $6.03 \pm 1.11$ & - & - & - & $6 \pm 1$ & $1 \pm 0.3$ & - & $0.7 \pm 0.4$ \\
VVDS020182331 & $19.85 \pm 1.41$ & - & - & - & $55 \pm 14$ & - & - & - \\
VVDS020147106 & $8.53 \pm 1.52$ & - & - & - & $22 \pm 6$ & $2.9 \pm 1$ & $1.4 \pm 0.6$ & - \\
VVDS020261328 & $10.20 \pm 2.01$ & - & - & - & $5 \pm 1$ & - & - & - \\
VVDS220596913 & $18.55 \pm 1.78$ & - & - & - & $33 \pm 6$ & $4.7 \pm 1$ & - & - \\
VVDS220584167 & $17.90 \pm 1.83$ & - & - & - & $48 \pm 10$ & $11 \pm 3$ & $6 \pm 3$ & $7 \pm 3$ \\
VVDS220544103 & $25.32 \pm 2.97$ & $38 \pm 9$ & $46 \pm 12$ & $114 \pm 14$ & $161 \pm 29$ & - & - & - \\
VVDS220015726 & $9.47 \pm 1.41$ & - & - & - & $26 \pm 3$ & $4.6 \pm 1$ & - & - \\
VVDS220014252 & $37.54 \pm 1.73$ & - & - & - & $212 \pm 27$ & $48 \pm 6$ & $15 \pm 2$ & - \\
\hline
\end{tabular}

${ }^{1}$ As measured in the optical VIMOS spectra.

Table 3. Metallicity $(12+\log (\mathrm{O} / \mathrm{H}))$ and stellar mass estimates for the 7 galaxies observed with SINFONI.

\begin{tabular}{cccccccc}
\hline \hline Galaxy & $Z_{\mathrm{PMC} 08}{ }^{1}$ & $Z_{\mathrm{PMC} 08 \text { cor. }^{2}}$ & $Z_{\mathrm{PMC} 08 \rightarrow \mathrm{T} 04^{3}}$ & $Z_{\mathrm{PMC} 08 \text { cor } \rightarrow \mathrm{T} 04^{3}}$ & $\log \left(M^{\star} / M_{\odot}\right)^{4}$ & $\log (\mathrm{N} / \mathrm{O})^{5}$ & $\log \left(\mathrm{SSFR} \mathrm{yr}{ }^{-1}\right)^{6}$ \\
\hline VVDS020116027 & $8.46 \pm 0.09$ & $8.43 \pm 0.27$ & $8.66 \pm 0.12$ & $8.62 \pm 0.37$ & $10.09_{-0.21}^{+0.38}$ & $-1.13 \pm 0.49$ & $-8.72 \pm 0.20$ \\
VVDS020147106 & $8.37 \pm 0.16$ & $8.15 \pm 0.16$ & $8.56 \pm 0.2$ & $8.29 \pm 0.19$ & $10.23_{-0.18}^{+0.01}$ & $-0.77 \pm 0.22$ & $-8.22 \pm 0.31$ \\
VVDS220596913 & $8.40 \pm 0.11$ & - & $8.59 \pm 0.14$ & - & $10.93_{-0.29}^{+0.12}$ & - & $-8.94 \pm 0.21$ \\
VVDS220584167 & $8.56 \pm 0.13$ & $8.44 \pm 0.24$ & $8.80 \pm 0.18$ & $8.64 \pm 0.33$ & $11.08_{-0.23}^{+0.28}$ & $-0.95 \pm 0.43$ & $-8.73 \pm 0.24$ \\
VVDS220544103 & - & - & - & - & $10.71_{-0.41}^{+0.16}$ & - & $-7.81 \pm 0.21$ \\
VVDS220015726 & $8.44 \pm 0.11$ & - & $8.63 \pm 0.13$ & - & $10.79_{-0.13}^{+0.29}$ & - & $-8.88 \pm 0.13$ \\
VVDS220014252 & $8.56 \pm 0.08$ & $8.20 \pm 0.12$ & $8.80 \pm 0.10$ & $8.35 \pm 0.15$ & $10.78_{-0.39}^{+0.07}$ & $-0.53 \pm 0.22$ & $-7.83 \pm 0.30$ \\
\hline
\end{tabular}

${ }^{1}$ Metallicity estimate using PMC08 indicator; ${ }^{2}$ metallicity estimate using PMC08 indicator and N/O correction with the sulfur lines; ${ }^{3}$ corresponding metallicities converted into T04 metallicity estimator; ${ }^{4}$ stellar mass in solar units (see Sect. 3.1$) ;{ }^{5} \log (\mathrm{N} / \mathrm{O})$ estimated with the N2S2 ratio; ${ }^{6}$ specific SFR in $\mathrm{yr}^{-1}$, derived with $L(\mathrm{H} \alpha)$.

detect $[\mathrm{N}$ II $] 6584$ for three galaxies). This led us to use the N2 indicator, defined as follows:

$\mathrm{N} 2=\log \frac{[\mathrm{N} \text { II }] 6584}{\mathrm{H} \alpha}$

which is subject to many calibrations for metallicity estimates (Kewley \& Dopita 2002; Denicoló et al. 2002; Nagao et al. 2006; Yin et al. 2006; Perez-Montero \& Contini 2009). In our cases, the N2 parameter offered two main advantages to derive metallicity. Due to the proximity of the $\mathrm{H} \alpha$ and [N II] $6584 \AA$ lines, the $\mathrm{N} 2$ ratio is independant of reddening correction and uncertainty in relative flux calibration. Recently, Perez-Montero \& Contini (2009) (hereafter PMC08) provided a new calibration of this parameter based on a sample of objects with a direct determination of the corresponding ionic abundances:

$12+\log \mathrm{O} / \mathrm{H}=9.07+0.79 \times \mathrm{N} 2$.

Nevertheless, this parameter presents a large scatter, which can be due to many processes, as explained in Pérez-Montero \& Díaz (2005). One possible explanation is the dependence of the calibration on the nitrogen-to-oxygen abundance ratio N/O. PMC08 investigated how the $\mathrm{N} 2$ abundance indicator can be altered by the value of the N/O ratio. They found the following deviation:

$\Delta(\mathrm{O} / \mathrm{H})=0.5 \log \mathrm{N} / \mathrm{O}+0.66$.

Following their method, we had to determine the nitrogen-tooxygen abundance ratio. Perez-Montero \& Contini (2009) propose an empirical calibration for it, derived from the same sample of H II regions they used to derive Eqs. (2) and (3). For our purpose, considering the emission lines we have in our spectra, we have used use the $\log \frac{[\mathrm{N} \text { II] } 6584}{[\mathrm{SI}] 6717,6731}=\mathrm{N} 2 \mathrm{~S} 2$ ratio leading to the following calibration:

$\log (\mathrm{N} / \mathrm{O})=1.26 \times \mathrm{N} 2 \mathrm{~S} 2-0.86$.
In this work, we first calculated the metallicities using Eq. (2): the results are listed in Table 3. Unfortunately, the measurement of the sulfur lines ([S II]6717, 6731 $\AA$ ) is very difficult, and we sometimes only had a measurement for one of the two lines, so we derived the other using the relation (5) which is valid if we assume a typical density of $100 \mathrm{~cm}^{-2}$ (Ramsbottom et al. 1993):

$\frac{[\mathrm{S} \text { II }] 6717}{[\mathrm{~S} \text { II }] 6731}=1.32$.

In Osterbrock (1974), Fig. 5.3 p. 134, we can see that at these typical densities, the value of the ratio changes by 0.1 at most. We were thus able to "correct" the metallicities of 4 galaxies, the ones for which we had a measurement of [S II]6717, $6731 \AA$. The derived metallicities, corrected for the N/O ratio, are also listed in Table 3.

For three galaxies (VVDS220544103, VVDS020261328 and VVDS020182331) we did not measure the [N II]6584 $\AA$ line. However, observations in the $J$ band were available for the first galaxy (VVDS220544103) enabling to measure [O III]4959, $5009 \AA$ and $\mathrm{H} \beta$ emission lines (see Table 2). With $\mathrm{H} \alpha$, measured in the $H$ band, and [O II] $3727 \AA$ (from the VIMOS spectrum) we had thus in hand five emission lines for this galaxy to derive metallicity using the $R_{23}$ indicator. The ratio of the Balmer lines $\mathrm{H} \alpha / \mathrm{H} \beta$ gives an estimate of the attenuation by the dust. Assuming a classical value for the intrinsic ratio $F(\mathrm{H} \alpha) / F(\mathrm{H} \beta)$ of 2.85, our measurements of the $\mathrm{H} \alpha$ and $\mathrm{H} \beta$ lines gave an extinction coefficient of $C=0.31 \pm 0.33$. We were then able to correct the emission-line fluxes for reddening using the Calzetti extinction law (Calzetti 2001) and use these values to derive metallicity. We obtained $\log R_{23}=0.702$ which led to an estimate of the metallicity of $12+\log (\mathrm{O} / \mathrm{H})=8.78$, using the calibration of Zaritsky et al. (1994). Kewley \& Ellison (2008) 
provide a way to translate this value to T04 (the calibration used in Tremonti et al. (2004)). We finally obtained $12+$ $\left.\log (\mathrm{O} / \mathrm{H})\right|_{\mathrm{T} 04}=8.74$ for $\operatorname{VVDS} 220544103$.

\section{The mass-metallicity relation}

\subsection{The $M Z$ relation}

Figure 2 shows the position of the seven galaxies at $z \sim 1.4$ in the $M Z$-plane (blue circles) using the metallicities and the stellar masses listed in Table 3. In order to compare with the $M Z$ relations previously derived at different redshifts, we also draw in this figure the $M Z$ relations obtained in the local Universe (Tremonti et al. 2004) as derived from the the SDSS, at $z \sim 0.7$ (Lamareille et al. 2009) and $z \sim 1$ (Pérez-Montero et al. 2009) from the VVDS sample, at $z \sim 1$ from zCOSMOS (Contini et al., in prep.), and at $z \sim 2$ (Erb et al. 2006). However, such a comparison requires great precautions, as pointed out in Kewley \& Ellison (2008). In their study, Kewley \& Ellison (2008) investigate the influence of different metallicity calibrations on the $M Z$ relation and showed that the absolute value of the metallicity can vary up to 0.8 dex depending on the calibration used. Taking this remark into consideration, our purpose being to compare the $M Z$ relation at $z \sim 1.4$ to the ones at others epochs, we had to be consistent with the calibrations used in other studies.

Firstly, at $z \sim 0$ Tremonti et al. (2004) used an indicator based on the Bayesian calibration CL01 (for Charlot \& Longhetti 2001) - we will refer as T04 - which make use of a large set of emission lines to derive metallicity. In order to translate our metallicities into the T04 calibrator, we first converted to Denicoló et al. (2002) N2 calibration and then applied the transformation given in Kewley \& Ellison (2008) to get T04 metallicities. If $x$ stands for the metallicity estimate of Perez-Montero $\&$ Contini (2009) and $y$ for that of T04, we then have:

$y=-0.217469 x^{3}+5.80493 x^{2}-50.2318 x+149.836$.

The resulting points in Fig. 2 are plotted as blue circles.

Secondly, at $z \sim 2$ Erb et al. (2006) used another calibration of the N2 indicator: PP04 (Pettini \& Pagel 2004). To be consistent we chose to convert their relation into T04. In Kewley \& Ellison (2008) we found the way to convert from PP04 to T04, as follows (if $x$ stands for the estimate of metallicity with PP04 and $y$ with T04):

$y=2.6766690 x^{3}-68.471750 x^{2}+585.1750 x-1661.9380$.

The resulting curve is shown in Fig. 2 in red. The three other relations (Lamareille et al. 2009; Pérez-Montero et al. 2009, Contini et al., in prep.) are already calibrated with T04 estimator.

While Tremonti et al. (2004), Erb et al. (2006), Lamareille et al. (2009) and Pérez-Montero et al. (2009) found a monotonic relation between the metallicity and stellar mass - more massive galaxies have a higher nebular metallicity than less massive ones - our small sample of galaxies at $z \sim 1.4$ does not show any obvious correlation between metallicity and stellar mass, even though one could imagine $Z$ to increase with stellar mass. Actually, if we fit a line through our 7 points, we obtain a positive slope $\left(12+\log (\mathrm{O} / \mathrm{H})=0.101 \log \left(M^{\star} / M_{\odot}\right)+7.61\right)$. The mean metallicity for this sample of seven galaxies is $12+$ $\log (\mathrm{O} / \mathrm{H})=8.62$ with a scatter of $0.22 \mathrm{dex}$. Note however that the $z \sim 1.4$ galaxies are lying below the $M Z$ relation derived in the local universe from SDSS data (blue line in Fig. 2). The shift in metallicity is roughly -0.3 dex, which clearly confirms the evolutionary trend of the galaxies metallicity with cosmic time

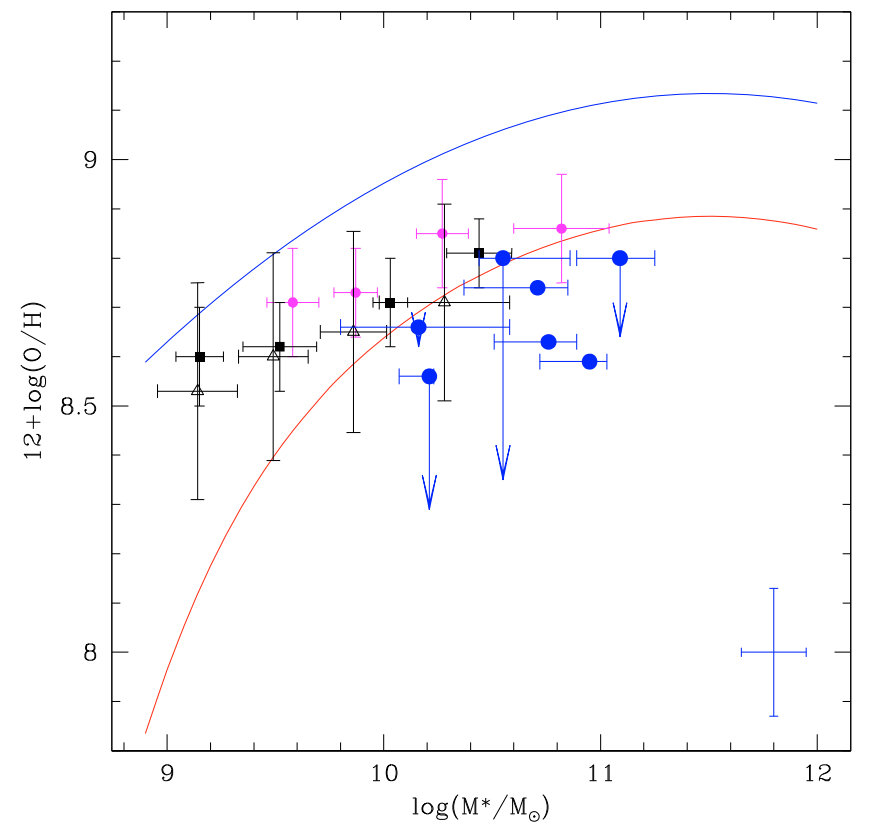

Fig. 2. Mass-metallicity relation for the sample of seven VVDS galaxies (blue circles) at $z \sim 1.4$. The arrows show how the metallicities change when we include the correction of $\log (\mathrm{N} / \mathrm{O})$, following Perez-Montero \& Contini (2009) prescription. The error bars at the bottom right corner show typical uncertainties of our data. The blue curve is the $M Z$ relation in the local universe as derived from the SDSS by Tremonti et al. (2004). The red curve is the $M Z$ relation at $z \sim 2$ by Erb et al. (2006). The $M Z$ relation at $z \sim 0.7$ (Lamareille et al. 2009) and $z \sim 1$ (Pérez-Montero et al. 2009) from the VVDS sample, and at $z \sim 1$ from zCOSMOS (Contini et al., in prep.) are shown in black open triangles, black squares, and magenta circles respectively. All metallicities have been translated into T04 to facilitate comparisons.

for a given stellar mass. Six out of seven galaxies have metallicities lower than the median $z \sim 2$ relation of Erb et al. (2006) which seems to draw an upper limit in metallicity for our sample. We can wonder why our galaxies seem poorly metallic, and in particular less metallic than Erb et al. (2006) relation.

Recently Ellison et al. (2008a) investigated the systematic effects of specific star formation rate (SSFR) on the $M Z$ relation. At a given mass, SDSS galaxies with high SSFR have in average lower metallicities than galaxies with low SSFR. We thus derived SSFR for the 7 galaxies in our sample, using the $\mathrm{H} \alpha$ luminosity, the SFR calibration by Argence \& Lamareille (2009) (Eq. (17)) and the stellar mass given by the SED fitting. To be able to compare with the entire VVDS sample, we also derived specific SFR for galaxies in the $22 \mathrm{~h}+02 \mathrm{~h}$ "wide" fields, for which we had a measure of $F([\mathrm{O}$ II $] 3727 \AA)$ and an estimate of the stellar mass. Argence \& Lamareille (2009) provide a calibration of the SFR based on the [O II] $3727 \AA$ line (Eq. (18)) which is consistent with the $\mathrm{H} \alpha$ calibration. In Fig. 3 we show the SSFR as a function of stellar mass for the full VVDS $22 \mathrm{~h}+02 \mathrm{~h}$ "wide" sample, together with our $z \sim 1.4$ VVDS galaxies. As one can see our galaxies lie on top of the mean distribution in SSFR, even out of the $1 \sigma$ limits (except for one), which could explain why their metallicity is so low. As a matter of fact our sample is clearly biased toward high specific star formation rates. Several explanations are proposed for this trend (see Ellison et al. 2008a, for more details): galactic winds could be more efficient in extracting metals at higher SSFRs, inflows of metal-poor gas could increase the SFR, etc. The fact that these seven galaxies show on 


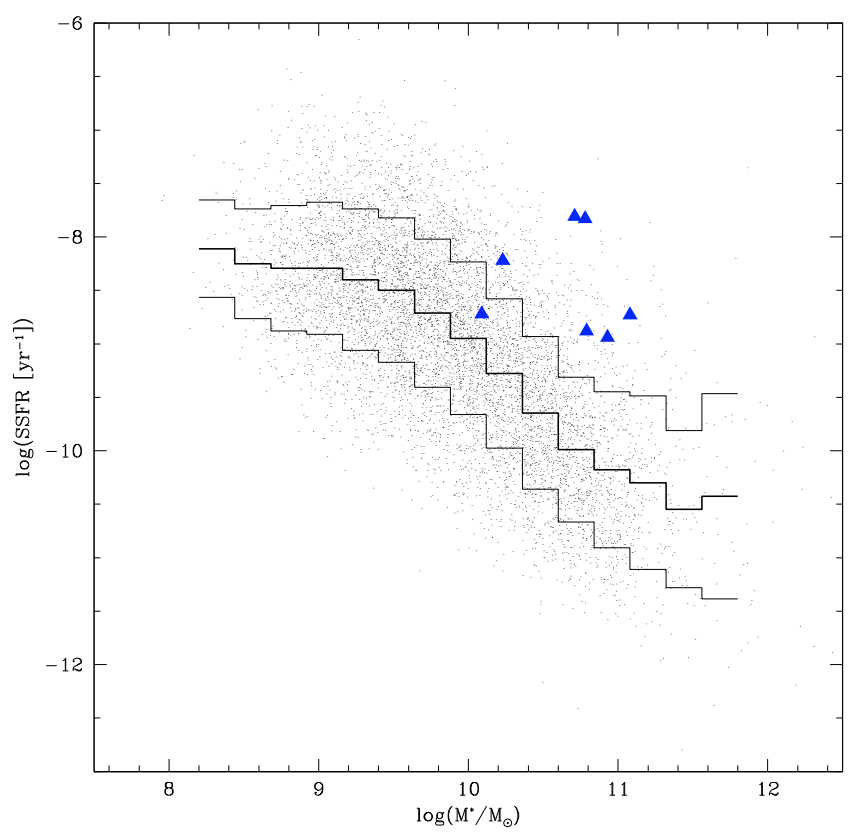

Fig. 3. Specific star formation rate vs. stellar mass for VVDS galaxies in the $02 \mathrm{~h}$ and $22 \mathrm{~h}$ fields (see Sect. 3.1 for mass estimates). Values are obtained with SFR derived from [O II] $3727 \AA$ luminosity, with the calibration of Argence \& Lamareille (2009). The histograms represent the mean specific SFR distribution (bold black line) and $1 \sigma$ scatter (thick black lines). The blue triangles stand for the seven galaxies of our sample, which SFR have been derived from $\mathrm{H} \alpha$ luminosity, as in Argence \& Lamareille (2009).

average higher SSFRs than the global VVDS sample is not surprising as they were selected on the basis of the intensity of the [O II] $3727 \AA$ line (see Sect. 2.1). This bias could explain why the $M Z$ relation we find at $z \sim 1.4$ seems shifted toward low metallicities. Another explanation, which could be linked to the SSFR one, has been recently proposed by Peeples et al. (2009). These authors claim that the presence of tidal interactions in majors mergers could possibly account for a fading in oxygen abundance as metal-poor gas may inflow from large radii. In our sample, VVDS220596913, VVDS220544103 and VDS020116027 seem indeed to undergo tidal interactions as revealed by their peculiar velocity fields (see Epinat et al. 2009).

\subsection{Influence of the $\mathrm{N} / \mathrm{O}$ ratio}

Figure 4 shows the relation between the stellar mass and the nitrogen-to-oxygen abundance ratio N/O for the SDSS starforming galaxies in the local universe (Perez-Montero \& Contini 2009, their Fig. 13). The four VVDS galaxies at $z \sim 1.4$ for which we were able to derive $\log (\mathrm{N} / \mathrm{O})$ are also shown in this figure as filled squares. Even if the dispersion around the relation defined by the SDSS sample is high, it is clear that the four VVDS galaxies are consistent with this relation. The influence of the nitrogen-to-oxygen abundance ratio on the metallicity is shown in Fig. 2 with the downwarding arrows. As noticed by Perez-Montero \& Contini (2009) the metallicities are significantly shifted to lower values for high N/O abundances ratios. This is especially the case for VVDS020147106 and VVDS220014252 which exhibit the highest N/O values. Looking at Fig. 2 we must keep in mind the important error bars going up to nearly 0.5 dex for the N/O ratio (see Table 3). Nevertheless, even if we take into account these errors, the

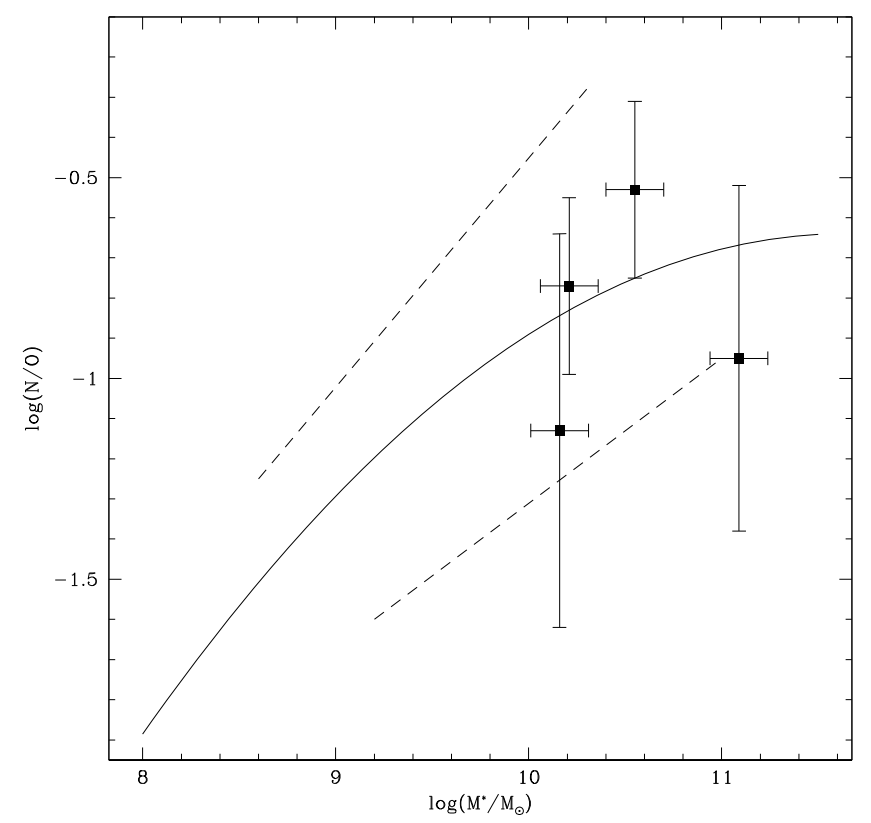

Fig. 4. Relation between stellar mass and the N/O abundance ratio. The solid line is the median relation of the SDSS star-forming galaxies in the local universe(see Perez-Montero \& Contini 2009, Fig. 14).The dashed lines indicate the scatter of this relation. The filled black squares correspond to the four VVDS galaxies for which we have measurements of the sulfur lines to derive N/O ratio.

downwarding shifts in metallicity - for VVDS020147106 and VVDS220014252 - are too important to deny any effective decline in oxygen abundance: indeed the drops in oxygen abundance are larger than the error bars themselves, which guarantee an effective decline.

\section{Conclusion and prospects}

We have determined the stellar mass and metallicity of seven VVDS star-forming galaxies at $z \sim 1.4$ observed in the NIR with the integral-field spectrograph SINFONI. Keeping in mind the uncertainties inherent to the metallicity and mass estimates, and considering also that our small sample does not allow to draw any firm conclusion, we can however draw the following points. Our sample of seven $z \sim 1.4$ galaxies shows if any, a mild correlation between metallicity and stellar mass $(\sim 0.1$ logarithmic slope). The mean metallicity is $12+\log (\mathrm{O} / \mathrm{H})=8.62$ with a scatter of 0.22 dex. All the galaxies in our sample are just under the relation of Erb et al. (2006) at $z \sim 2$, except for one galaxy that lies just above. The low metallicity measured in these galaxies at $z \sim 1.4$ could be due to their high specific star formation rate and/or to the tidal interactions allowing for inflow of metalpoor gas in the cases of VVDS220596913, VVDS220544103 and VDS020116027. Accounting for the dependence of oxygen abundance estimate on the $\mathrm{N} / \mathrm{O}$ abundance ratio, as suggested by Perez-Montero \& Contini (2009), would lower significantly the metallicity for two galaxies showing the largest N/O abundance ratios. When completed, the MASSIV survey will gather a representative sample of roughly 80 galaxies observed with SINFONI within $1<z<2$. It will allow to shed new light on these issues enabling to probe the dynamical and physical properties of these galaxies. Concerning the $M Z$ relation, a statistical study will be undertaken over a large range of stellar masses, computing median metallicities in bins of mass and deriving the median relation between $12+\log (\mathrm{O} / \mathrm{H})$ and $M^{\star}$ and its 
intrinsic scatter. Comparing these observations to the predictions of galaxy evolution models (e.g. de Rossi et al. 2007) will also be an major issue to investigate within MASSIV.

Acknowledgements. We wish to thank the ESO staff at Paranal Observatory and especially the SINFONI team at VLT for their support during observations. We also thank the referee for the constructive comments which help to improve the quality of this paper. This work has been partially supported by the CNRS-INSU Programme National de Galaxies and by the french ANR grant ANR-07-JCJC-0009.

\section{References}

Argence, B., \& Lamareille, F. 2009, A\&A, 495, 759

Arnouts, S., Walcher, C. J., Le Fèvre, O., et al. 2007, A\&A, 476, 137

Calzetti, D. 2001, PASP, 113, 1449

Charlot, S., \& Longhetti, M. 2001, MNRAS, 323, 887

Cooper, M. C., Tremonti, C. A., Newman, J. A., \& Zabludoff, A. I. 2008, MNRAS, 390, 245

Cowie, L. L., \& Barger, A. J. 2008, ApJ, 686, 72

De Lucia, G., Kauffmann, G., \& White, S. D. M. 2004, MNRAS, 349, 1101

de Rossi, M. E., Tissera, P. B., \& Scannapieco, C. 2007, MNRAS, 374, 323

Denicoló, G., Terlevich, R., \& Terlevich, E. 2002, MNRAS, 330, 69

Drory, N., Salvato, M., Gabasch, A., et al. 2005, ApJ, 619, L131

Eisenhauer, F., Abuter, R., Bickert, K., et al. 2003, in SPIE Conf. Ser. 4841, ed. M. Iye, \& A. F. M. Moorwood, 1548

Ellison, S. L., Patton, D. R., Simard, L., \& McConnachie, A. W. 2008a, ApJ, 672, L107

Ellison, S. L., Patton, D. R., Simard, L., \& McConnachie, A. W. 2008b, AJ, 135, 1877

Epinat, B., Contini, T., Le Fevre, O., et al. 2009, A\&A, 504, 789

Erb, D. K., Shapley, A. E., Pettini, M., et al. 2006, ApJ, 644, 813

Finlator, K., \& Davé, R. 2008, MNRAS, 385, 2181

Franzetti, P., Scodeggio, M., Garilli, B., Fumana, M., \& Paioro, L. 2008, in Astronomical Data Analysis Software and Systems XVII, ed. R. W. Argyle, P. S. Bunclark, \& J. R. Lewis, ASP Conf. Ser., 394, 642

Gallazzi, A., Charlot, S., Brinchmann, J., White, S. D. M., \& Tremonti, C. A. 2005, MNRAS, 362, 41

Garnett, D. R., Shields, G. A., Skillman, E. D., Sagan, S. P., \& Dufour, R. J. 1997, ApJ, 489, 63

Gavazzi, G., Bonfanti, C., Sanvito, G., Boselli, A., \& Scodeggio, M. 2002, ApJ, 576,135
Hayashi, M., Motohara, K., Shimasaku, K., et al. 2009, ApJ, 691, 140 Kewley, L. J., \& Dopita, M. A. 2002, ApJ, 142, 35

Kewley, L. J., \& Ellison, S. L. 2008, ApJ, 681, 1183

Lamareille, F., Mouhcine, M., Contini, T., Lewis, I., \& Maddox, S. 2004, MNRAS, 350, 396

Lamareille, F., Contini, T., Brinchmann, J., et al. 2006, A\&A, 448, 907

Lamareille, F., Brinchmann, J., Contini, T., et al. 2009, A\&A, 495, 53

Le Fèvre, O., Vettolani, G., Garilli, B., et al. 2005, A\&A, 439, 845

Lequeux, J., Peimbert, M., Rayo, J. F., Serrano, A., \& Torres-Peimbert, S. 1979, A\&A, 80, 155

Liang, Y. C., Hammer, F., \& Flores, H. 2006, A\&A, 447, 113

Lilly, S. J., Le Fevre, O., Hammer, F., \& Crampton, D. 1996, ApJ, 460, L1

Liu, X., Shapley, A. E., Coil, A. L., Brinchmann, J., \& Ma, C.-P. 2008, ApJ, 678, 758

Madau, P., Ferguson, H. C., Dickinson, M. E., et al. 1996, MNRAS, 283, 1388

Maier, C., Lilly, S. J., Carollo, C. M., Stockton, A., \& Brodwin, M. 2005, ApJ, 634,849

Maier, C., Lilly, S. J., Carollo, C. M., et al. 2006, ApJ, 639, 858

Maiolino, R., Nagao, T., Grazian, A., et al. 2008, A\&A, 488, 463

Mannucci, F., Cresci, G., Maiolino, R., et al. 2009, MNRAS, 398, 1915

Michel-Dansac, L., Lambas, D. G., Alonso, M. S., \& Tissera, P. 2008, MNRAS, 386, L82

Mouhcine, M., Baldry, I. K., \& Bamford, S. P. 2007, MNRAS, 382, 801

Nagao, T., Maiolino, R., \& Marconi, A. 2006, A\&A, 459, 85

Osterbrock, D. E. 1974, Astrophysics of Gaseous Nebulae (W. H. Freeman and Company)

Panter, B., Jimenez, R., Heavens, A. F., \& Charlot, S. 2008, MNRAS, 391, 1117

Peeples, M. S., Pogge, R. W., \& Stanek, K. Z. 2009, ApJ, 695, 259

Pérez-González, P. G., Rieke, G. H., Egami, E., et al. 2005, ApJ, 630, 82

Perez-Montero, E., \& Contini, T. 2009, MNRAS, 398, 949

Pérez-Montero, E., \& Díaz, A. I. 2005, MNRAS, 361, 1063

Pérez-Montero, E., Contini, T., Lamareille, F., et al. 2009, A\&A, 495, 73

Pettini, M., \& Pagel, B. E. J. 2004, MNRAS, 348, L59

Pozzetti, L., Bolzonella, M., Lamareille, F., et al. 2007, A\&A, 474, 443

Ramsbottom, C. A., Bell, K. L., \& Berrington, K. A. 1993, J. Phys. B Atom. Mol. Phys., 26, 4399

Savaglio, S., Glazebrook, K., Le Borgne, D., et al. 2005, ApJ, 635, 260

Saviane, I., Ivanov, V. D., Held, E. V., et al. 2008, A\&A, 487, 901

Shapley, A. E., Coil, A. L., Ma, C.-P., \& Bundy, K. 2005, ApJ, 635, 1006

Skillman, E. D., Kennicutt, R. C., \& Hodge, P. W. 1989, ApJ, 347, 875

Tremonti, C. A., Heckman, T. M., Kauffmann, G., et al. 2004, ApJ, 613, 898

Tresse, L., Ilbert, O., Zucca, E., et al. 2007, A\&A, 472, 403

Yin, S. Y., et al. 2006, A\&A

Zaritsky, D., Kennicutt, Jr., R. C., \& Huchra, J. P. 1994, ApJ, 420, 87 